\title{
Application of the linear matching method to creep-fatigue failure analysis of cruciform weldment manufactured of the austenitic steel AISI type $316 \mathrm{~N}(\mathrm{~L})$
}

\author{
Yevgen Gorash and Haofeng Chen \\ Department of Mechanical \& Aerospace Engineering, University of Strathclyde, James Weir Building, \\ 75 Montrose Street, Glasgow G1 1XJ, UK
}

Keywords: creep, damage, finite element analysis, FSRF, low-cycle fatigue, type 316 steel, weldment

\section{Introduction}

This paper demonstrates the recent extension of the Linear Matching Method (LMM) to include cyclic creep assessment [1] in application to a creep-fatigue analysis of a cruciform weldment made of the stainless steel AISI type $316 \mathrm{~N}(\mathrm{~L})$. The obtained results are compared with the results of experimental studies implemented by Bretherton et al. [2] with the overall objective to identify fatigue strength reduction factors (FSRF) of austenitic weldments for further design application. These studies included a series of strain-controlled tests at $550^{\circ} \mathrm{C}$ with different combinations of reversed bending moment and dwell time $\Delta t$. Five levels of reversed bending moment histories corresponding to defined values of total strain range $\Delta \varepsilon_{\text {tot }}$ in remote parent material $(1 \%, 0.6 \%, 0.4 \%, 0.3 \%, 0.25 \%)$ were used in combination with three variants of creep-fatigue conditions: pure fatigue, 1 hour and 5 hours of dwell period $\Delta t$ of hold in tension. An overview of previous works devoted to analysis and simulation of these experiments [2] and highlight of the LMM development progress could be found in [3].

Recently [1] the LMM has been much improved both theoretically and numerically including more accurate predictions of the stabilised cyclic response of a structure under creep conditions, and more accurate assessments of the resulting cyclic and residual stresses, creep strain, plastic strain range, ratchet strain and the elastic follow-up factor. Previously, Ponter and Chen [4] applied the earlier version of the LMM for the description of elastic, plastic and creep material behaviour to the numerical assessments of a cruciform weldment using the R5 standard creep-fatigue model according to the methodology of the life assessment Procedure R5 [5]. Those results [4] were acceptable, but not perfect in sense of agreement with corresponding experiments [2].

In fact, the analyses presented in this paper revisit previous LMM assessments [4] of the same experimental studies [2] using the improved method [1], more accurate modelling of the weld structure and the material behaviour of its regions including LCF endurance, creep and long-term strength properties. In contrast to [4], creep damage is assessed using time fraction rule instead of ductility exhaustion recommended in R5 Procedure [5], which provides over-conservatism in combination with time-hardening creep law. The non-linear creep-fatigue interaction diagram instead of linear is also found to be more suitable for the assessment of critical creep-fatigue damage.

\section{Structural model}

The geometry of the weldment specimen, shown schematically in Fig.1a, is reconstructed from [2] based on the given sketches of the cruciform weldment and the Manual Metal Arc (MMA) welding procedure. A continuous plate of width $200 \mathrm{~mm}$ and length of $1.8 \mathrm{~m}$ is divided, at its centre, into two parts, each of which is welded to the surface of a third plate of length $100 \mathrm{~mm}$. The weld area is subdivided into 3 regions: the parent material, assumed to be uniform away from the weld; the weld metal, deposited material during multi-pass welding process; and the heat-affected zone (HAZ), a thin layer between the weld and parent material. These regions are expected to have different mechanical properties including elasticity, plasticity, fatigue and creep, caused by microstructural transformations during the multi-pass welding process.

The FE-mesh for a 2D symmetric model of the specimen assumes a plane strain conditions since the specimen width $(200 \mathrm{~mm})$ is almost by an order of magnitude greater than the specimen thickness $(26 \mathrm{~mm})$ according to Fig.1a. The FE-mesh includes 5 separate areas with different material properties denoted in Fig.1b. Introduction of 2 additional material types (material without creep and totally elastic material) with reduced sets of parent material properties in the location of bending moment application avoids excessive stress concentrations in ratcheting and creep analysis. The FE-model consists of 977 finite elements of type CPE8R: 8-node biquadratic plane strain quadrilaterals with reduced integration. 


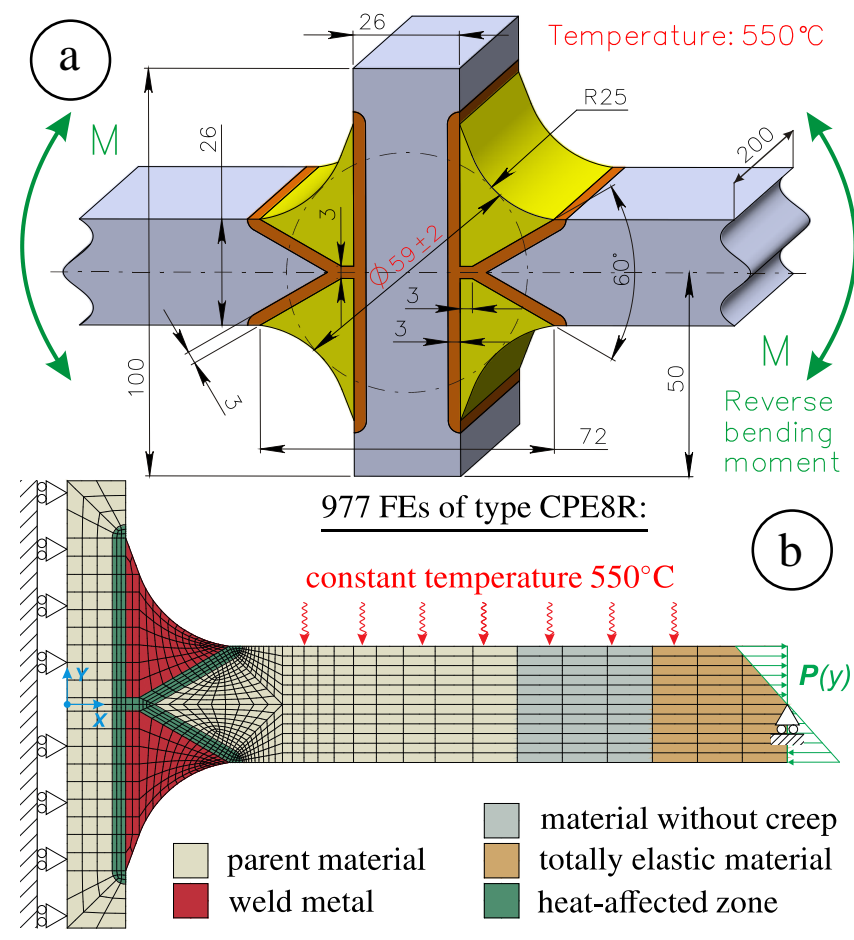

Figure 1: Geometrical and analysis parameters of the cruciform weld specimens: a) dimensions and applied loading; b) FE-mesh with designation of different materials, boundary conditions and mechanical loading
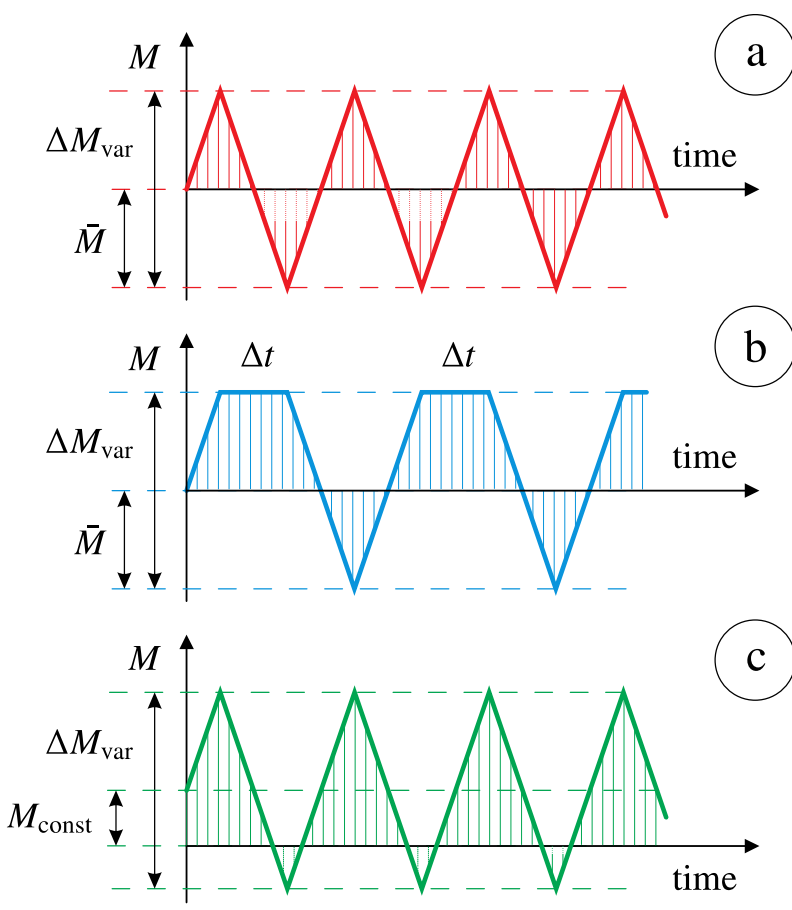

Figure 2: Assumed schematic loading history for the bending moment $M$ in: a) fully-reversed pure fatigue tests; b) fully-reversed creep-fatigue tests with dwells $\Delta t$ in tension; c) non-symmetric pure fatigue tests

For the purpose of shakedown and creep analysis using LMM, the conversion from straincontrolled test conditions to force-controlled loading in simulations has been carried out. Although the gradual increase of applied loading during the initial cycles [2] demonstrate significant cyclic hardening effects of the specimen material behaviour, which is typical for the steel AISI type 316N(L), such a simplification is valid considering that saturated cyclic structural response is dominant during the whole duration of tests. Therefore, in numerical simulations the arms of the specimen are subjected to 3 variants of bending moment history illustrated schematically in Fig.2. Pure fatigue analysis assumes a rapid reversal of bending moment of magnitude $\Delta M_{\mathrm{var}}$ as shown in Fig. 2a. Creep-fatigue analysis assumes a rapid reversal of bending moment of magnitude $\Delta M_{\mathrm{var}}$ separated by dwell periods of duration $\Delta t$ when the moment is maintained constant at $\bar{M}=\Delta M_{\mathrm{var}} / 2$ as shown in Fig.2b. Shakedown analysis assumes compound bending moment consisting of variable component of magnitude $\Delta M_{\mathrm{var}}$ and constant shift of value $M_{\text {const, }}$ hereby forming a load space as shown in Fig.2c.

The bending moment $M$ is applied through the linear distribution of normal pressure $P$ over the section of plate as shown in Fig. $1 \mathrm{~b}$ with the area moment of inertia $I_{\mathrm{x}}$ in regard to horizontal axis X:

$$
P(y)=M \cdot y / I_{X} \quad \text { with } I_{X}=a \cdot b^{3} / 12,
$$

where the width of plate $a=200 \mathrm{~mm}$, the thickness of plate $b=26 \mathrm{~mm}$, and $y$ is a vertical coordinate of plate section assuming the coordinate origin in the mid-surface.

\section{Material models and constants}

Mechanical properties of the materials composing cruciform weldment manufactured of the steel AISI type $316 \mathrm{~N}(\mathrm{~L})$ include the following material behaviour models and constants at $550^{\circ} \mathrm{C}$.

The conventional Ramberg-Osgood equation for the cyclic stress-strain curve, showing a smooth elastic-plastic transition, implemented in LMM code for the creep-fatigue analysis is following

$$
\Delta \varepsilon_{\text {tot }} / 2=\Delta \sigma /(2 \bar{E})+[\Delta \sigma /(2 B)]^{1 / \beta} \text { with } \quad \bar{E}=3 E /[2(1+v)]
$$

where $\Delta \varepsilon_{\text {tot }}$ is the total strain range; $\Delta \sigma$ is the total stress range in $\mathrm{MPa} ; B$ and $\beta$ are plastic material constants; $\bar{E}$ is the effective elastic modulus in MPa defined using the Young's modulus $E$ in MPa and the Poisson's ratio $v=0.3$, which are the elastic properties used in both R-O and EPP models.

The dependence of total strain range $\Delta \varepsilon_{\text {tot }}$ in $\%$ on the number of cycles to pure LCF failure $N^{*}$ is usually defined by a quadratic polynomial function [6] for S-N diagrams as follows 
$\log \left(\Delta \varepsilon_{\text {tot }}\right)=m_{0}+m_{1} \log \left(N^{*}\right)+m_{2} \log \left(N^{*}\right)^{2} \Leftrightarrow \log \left(N^{*}\right)=\frac{-m_{1}-\sqrt{m_{1}^{2}-4 m_{2}\left[m_{0}-\log \left(\Delta \varepsilon_{\text {tot }}\right)\right]}}{2 m_{2}}$,

where the coefficients of polynomial (3) defined by fitting the R66 endurance curves for parent and weld material of the steel AISI type $316 \mathrm{~N}(\mathrm{~L})$ at $550^{\circ} \mathrm{C}$ reported in [6] have the following values: $m_{0 \mathrm{p}}=1.73339, m_{1 \mathrm{p}}=-0.72959, m_{2 \mathrm{p}}=0.06170$ and $m_{0 \mathrm{w}}=1.85169, m_{1 \mathrm{w}}=-0.76094, m_{2 \mathrm{w}}=0.05951$.

The primary creep strain is described by the conventional "time hardening" form of power-law model or the Norton-Bailey equation, which has the following uniaxial form:

$$
\dot{\varepsilon}^{\text {cr }}=A \sigma^{n} t^{m} \quad \text { or } \quad \varepsilon^{\text {cr }}=[A /(m+1)] \sigma^{n} t^{m+1},
$$

where $\dot{\varepsilon}^{\text {cr }}$ is the creep strain, $\sigma$ is the applied stress in MPa, $t$ is the time in hours; $A, n$ and $m$ are the creep constants identified by fitting Eq.(4) to the primary stage of experimental creep curves [2].

The time to creep rupture $t^{*}$ dependent on stress $\sigma$ is described by the reverse power-law:

$$
t^{*}=B \sigma^{-k} \text {, }
$$

where $B$ and $k$ are the creep constants identified by fitting Eq.(5) to the experimental creep rupture data $[2,7]$. The whole set of material parameters for different weld zones is reported in Tab.1. It should be noted that the constants corresponding to elasticity and saturated cyclic plasticity were taken from [2]. The constants corresponding to primary creep strain and creep rupture of parent and weld material were identified employing the least squares method, while those constants for the HAZ were identified by logarithmic $(A, B)$ and simple $(n, m, k)$ averaging of parent and weld properties.

Table 1: Material parameters for the steel AISI type $316 \mathrm{~N}(\mathrm{~L})$ at $550^{\circ} \mathrm{C}$ fitting the experimental data [2, 7]

\begin{tabular}{l|l|lll|lll|ll}
\hline \multirow{2}{*}{ Zone } & Elastic & \multicolumn{2}{|c|}{ Saturated cyclic plasticity } & \multicolumn{3}{c|}{ Primary creep strain } & \multicolumn{2}{c}{ Creep rupture } \\
\cline { 2 - 11 } & $E(\mathrm{MPa})$ & $B(\mathrm{MPa})$ & $\beta$ & $\sigma_{\mathrm{y}}(\mathrm{MPa})$ & $\mathrm{A}\left(\mathrm{MPa}^{-n} / \mathrm{h}^{m+1}\right)$ & $\mathrm{n}$ & $\mathrm{m}$ & $\mathrm{B}\left(\mathrm{MPa}^{k} \mathrm{~h}\right)$ & $\mathrm{k}$ \\
\hline Parent & 160000 & 1741.96 & 0.29960 & 270.662 & $6.604 \mathrm{E}-19$ & 5.769 & -0.55 & $2.172 \mathrm{E}+26$ & 8.927 \\
Weld & 122000 & 578.99 & 0.10162 & 307.894 & $6.597 \mathrm{E}-23$ & 7.596 & -0.5 & $5.993 \mathrm{E}+29$ & 10.61 \\
HAZ & 154000 & 1632.31 & 0.25304 & 338.731 & $6.600 \mathrm{E}-21$ & 6.683 & -0.525 & $1.291 \mathrm{E}+28$ & 9.768 \\
\hline
\end{tabular}

\section{Structural integrity assessments}

The design limits were evaluated with an elastic-perfectly-plastic (EPP) model and a von Mises yield condition using material constants $\left(E, \sigma_{\mathrm{y}}\right.$ and $\left.v\right)$ reported in Tab.1, the history of bending moment according to Fig.2c, and the LMM [8] capable of upper and lower ratchet limit identification. The limit moment and shakedown limit have the following values respectively: $M_{\text {lim }}=10559430(\mathrm{~N} \mathrm{~mm})$ and $\Delta M_{\mathrm{sh}}=13614160(\mathrm{~N} \mathrm{~mm})$. The normalised moment is defined as the relation of variable moment range to shakedown limit: $\tilde{M}=\Delta M_{\mathrm{var}} / \Delta M_{\mathrm{sh}}$, where $\tilde{M}_{\mathrm{max}}=\Delta M_{\mathrm{lim}} / \Delta M_{\mathrm{sh}}=1.55124$. Hereby, for the specific case of cruciform weldment specimen (see Fig. 1a) the design limits are $\tilde{M} \in[0.5 \ldots 1.55124]$. The corresponding Bree interaction diagram and other details could be found in [3].

The basic routine of the proposed evaluation procedure for creep-fatigue damage assessment of cruciform weldments is the same as of the PNC time fraction procedure [9] developed by the Power Reactor and Nuclear Fuel Development Corporation (Ibaraki, Japan). The key differences between the PNC procedure and the proposed procedure are indicated in [3].

The general concept of the proposed creep-fatigue evaluation procedure considering time fraction rule for creep-damage assessment is illustrated on Fig.3 and consists of 5 steps.

Step 1: Saturated hysteresis loop. This step involves inelastic FEA using LMM in CAE-system ABAQUS incorporating FORTRAN user material subroutine UMAT, which includes implementation of Ramberg-Osgood model (2) and primary creep model (4) with material constants from Tab.1. The modification of the original LMM code [1] implemented in this work comprises the conversion from EPP model to R-O model, which provides more reasonable description of stress-strain response. The most important outputs for further creep-fatigue evaluation are $\Delta \varepsilon_{\text {tot }}$, stress $\sigma_{1}$ in the beginning of dwell period and elastic follow-up factor $Z=\bar{E} \varepsilon^{\mathrm{cr}} / \Delta \sigma_{\mathrm{cr}}$.

Step 2: Fatigue damage. This step is based upon the $\Delta \varepsilon_{\text {tot }}$ identified in Step 1 and polynomial function (3) for S-N diagrams characterising LCF properties. The fatigue damage accumulated per 1 cycle is calculated using the value of $N^{*}$ derived from R66 fatigue endurance curves [6]. 
Step 3: Stress relaxation. This step is based upon the relaxation problem with elastic follow-up, which has the analytical solution for stress function in case of "time-hardening" creep model (4):

$\frac{d \varepsilon^{\mathrm{cr}}}{d t}+\frac{Z}{\bar{E}} \frac{d \sigma}{d t}=0 \Rightarrow \sigma\left(t, Z, \sigma_{1}\right)=\left[\sigma_{1}^{1-n}-\frac{t^{m+1} \bar{E} A(1-n)}{Z(m+1)}\right]^{1 /(1-n)} \quad$ and $\quad \varepsilon^{\mathrm{cr}}=\left[\sigma_{1}-\sigma\left(t, Z, \sigma_{1}\right)\right] \frac{Z}{\bar{E}}$,

where $Z$ and $\sigma_{1}$ are taken from Step 1; $A, n$ and $m$ are creep constants for Eq. (4) from Tab.1.

The average stress $\bar{\sigma}$ over the dwell period $\Delta t$ is defined numerically as a mean value of the nonintegrable function $\sigma\left(t, Z, \sigma_{1}\right)$ on some closed interval $t \in[0 \ldots \Delta t]$, as shown in Fig.3.

Step 4: Creep damage. This step is based upon the $\bar{\sigma}$ identified in Step 3 and creep rupture curves (5) characterising creep endurance properties. The creep damage accumulated per 1 cycle is calculated considering time fraction rule and using the experimental creep rupture data $[2,7]$.

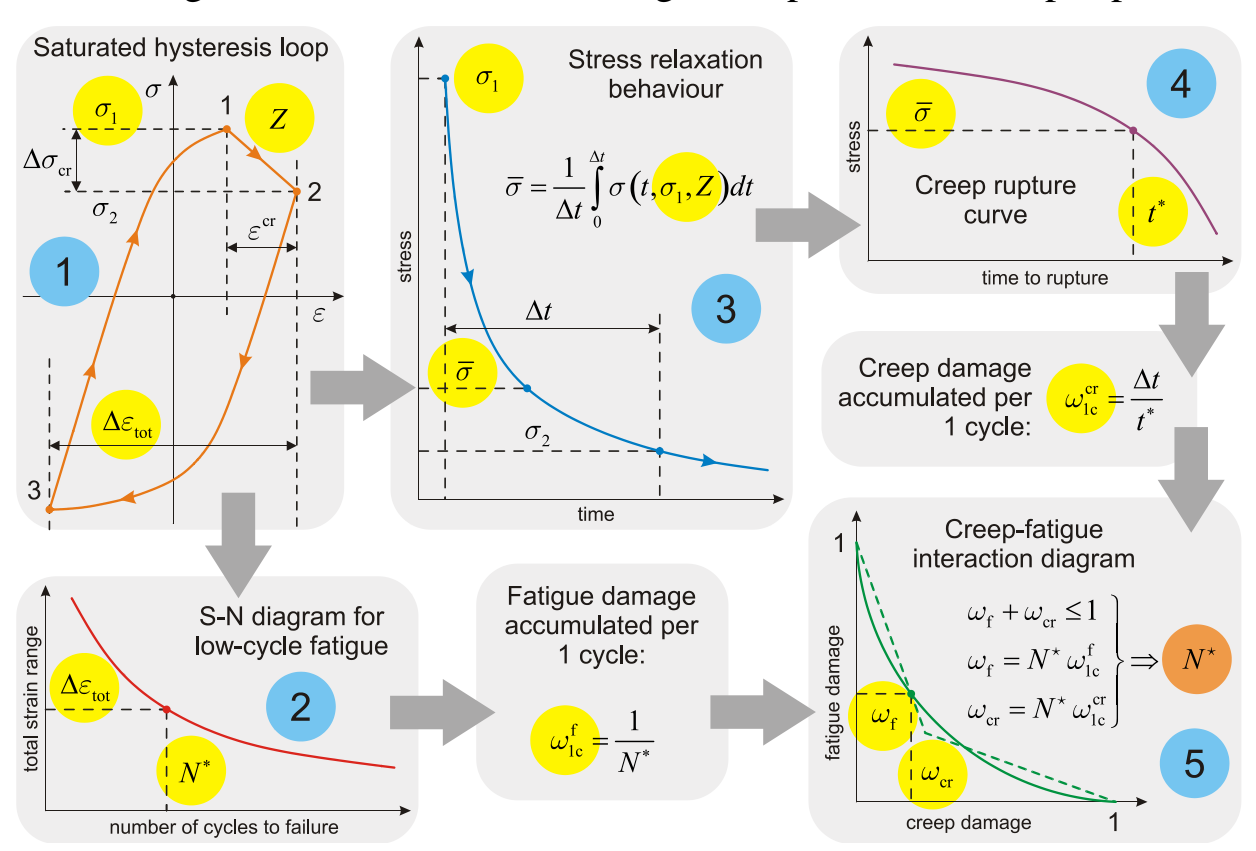

Figure 3: The general concept of creep-fatigue evaluation procedure considering time fraction rule for creep-damage assessment based on the PNC procedure [9]
Step 5: Creep-fatigue interaction. This step is based upon the values of total fatigue $\omega_{\mathrm{f}}$ and creep $\omega_{\text {cr }}$ damage, where the values of $\omega_{1 \mathrm{c}}^{\mathrm{f}}$ and $\omega_{1 \mathrm{c}}^{\mathrm{cr}}$ are taken from Step 2 and Step 4 respectively. The output of evaluation procedure $N^{\star}$ (number of cycles to failure under creep-fatigue interaction conditions) is usually defined employing the damage interaction diagrams [10], as shown in Fig.3. The most commonly used types of damage diagrams are described in $[3,10]$. In the formulation of this procedure, a novel approach to construct the non-linear damage diagram proposed by Skelton and Gandy [10] assuming "creep-fatigue" and "fatigue-creep" damage interactions was found the most suitable in combination with time fraction rule:

$$
\frac{\omega_{\mathrm{f}}}{1-\omega_{\mathrm{cr}}}+\frac{\omega_{\mathrm{cr}}}{1-\omega_{\mathrm{f}}}=1 \Rightarrow \begin{aligned}
& a N^{\star 2}-b N^{\star}+1=0, \\
& N^{\star}=\left(-b-\sqrt{b^{2}-4 a c}\right) /(2 a),
\end{aligned} \quad \text { where } \begin{aligned}
& a=\left[\omega_{1 \mathrm{c}}^{\mathrm{cr}}\right]^{2}+\left[\omega_{1 \mathrm{c}}^{\mathrm{f}}\right]^{2}+\omega_{\mathrm{lc}}^{\mathrm{cr}} \omega_{1 \mathrm{c}}^{\mathrm{f}} \\
& b=2 \omega_{1 \mathrm{c}}^{\mathrm{cr}}+2 \omega_{1 \mathrm{c}}^{\mathrm{f}},
\end{aligned}
$$

where the key parameter $N^{\star}$ is derived by solving the quadratic equation. This diagram provides a reasonable approximation to the well-known ASME bi-linear diagram with intersection of $(0.3,0.3)$.

\section{Validation and extrapolation of results}

The experimental studies of cruciform weldment [2] have been simulated employing the FEA with the LMM based upon FE-model and loading conditions described in Sect.2 and the material models described in Sect.3. The outputs of the LMM have been processed by the proposed creep-fatigue evaluation procedure described in Sect.4. The 5 variants of normalised moment $\tilde{M}$ used in the FEA and their correspondence to $\Delta \varepsilon_{\text {tot }}$ in tests are defined numerically as $1.4705-1,1.1538-0.6,0.9255-$ $0.4,0.7774-0.3,0.691-0.25$. The following function similar to the R-O model (2) is formulated:

$$
\Delta \varepsilon_{\text {tot }}(\tilde{M})=p_{1} \tilde{M}+p_{2} \tilde{M}^{p_{3}},
$$

where $p_{1}=0.2817, p_{2}=0.17649$ and $p_{3}=3.11051$ are the fitting parameters corresponding to the particular geometry of the parent material plate shown in Fig.1a.

The complete summary of experimental and corresponding simulation conditions as well as outputs in the form of number of cycles to failure $N^{\star}$ and location of failure is reported in [3]. The 
ratio between the fatigue $\omega_{\mathrm{f}}$ and creep $\omega_{\text {cr }}$ components of the total damage indicates that with $\Delta t=1$ hour the fatigue damage is dominant, whereas when $\Delta t$ is increased to 5 hours creep damage becomes dominant. Visual comparison of the observed and predicted $N^{\star}$ in Fig. 4 for 3 variants of dwell period $\Delta t$ shows that 9 of the 11 simulations accurately predict the experimental results.

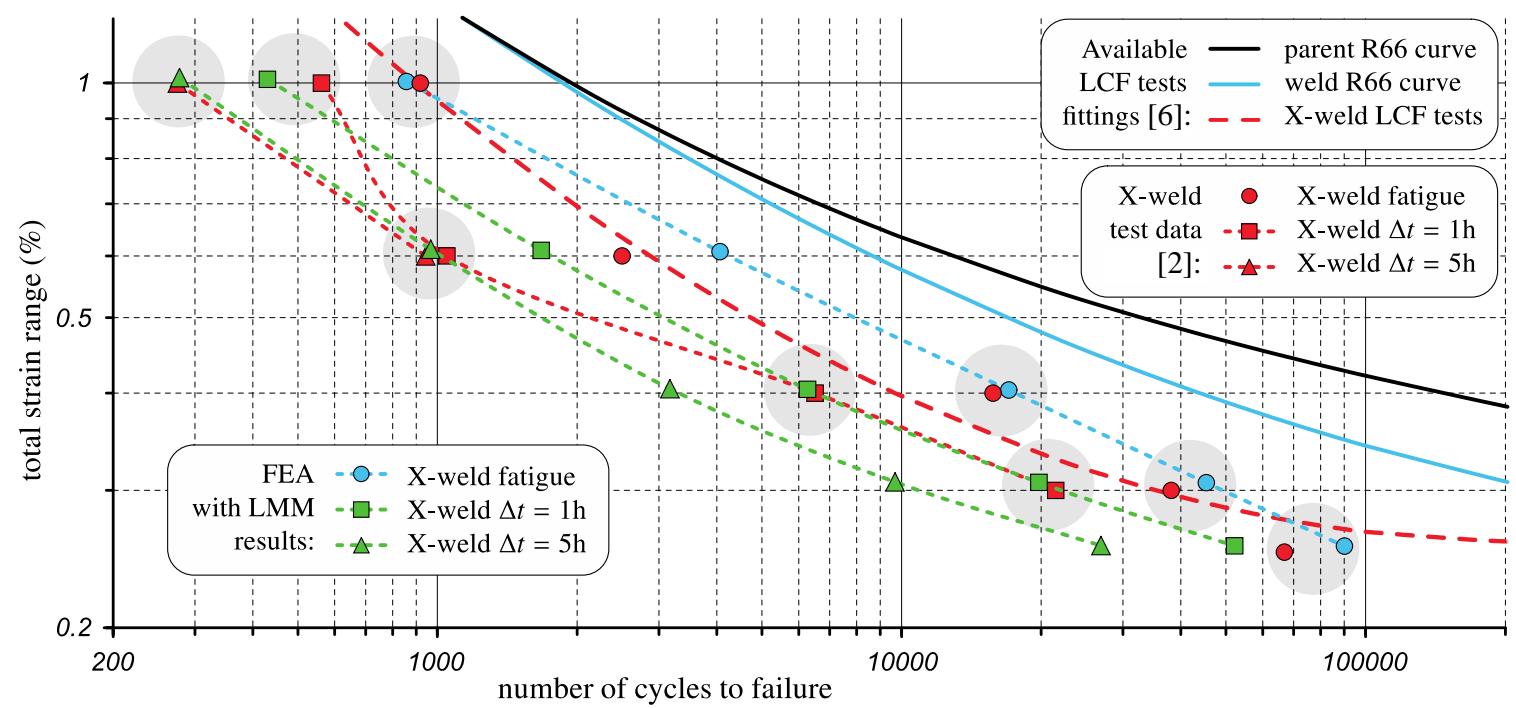

Figure 4: Results of creep-fatigue assessment in application to cruciform weldment and comparison with experiments [2]

Since the proposed approach is successfully validated against experimental data (see Fig.4), it can be used for the formulation of an analytic assessment model suitable for the fast estimation of $N^{\star}$ for a variety of loading conditions. The low computational effort required by the LMM compared to other computational techniques makes it possible and relatively easy to extrapolate numerical predictions for loading conditions not captured by the available experiments [2]. This extrapolation comprises the extension of the $\Delta t$ duration up to 10000 hours and a number of additional FE-simulations with LMM. The whole array of obtained results reported in [3] for the same set of applied $\tilde{M}$ is fitted using the least squares method by the following power-law function for $N^{\star}$ dependent on arguments $\tilde{M}$ and $\Delta t$ :

$$
\log \left(N^{\star}\right)=a(\Delta t) \tilde{M}^{-b(\Delta t)} \quad \text { with } \quad a(\Delta t)=a_{1} \log (\Delta t+1)+a_{2} \quad \text { and } \quad b(\Delta t)=b_{1} \log (\Delta t+1)+b_{2},
$$

where the independent fitting parameters: $a_{1}=-0.4921, a_{2}=3.708929, b_{1}=0.0255, b_{2}=0.754959$.

Having defined $N^{\star}$ by Eq.(9), the residual service life in years is therefore dependent on the duration of 1 cycle, which consists of dwell period $\Delta t$ and relatively short time of deformation:

$$
L^{\star}=N^{\star}\left[\Delta t /(365 \cdot 24)+2 \Delta \varepsilon_{\mathrm{tot}}(\tilde{M}) /(\dot{\varepsilon} \cdot 365 \cdot 24 \cdot 60 \cdot 60)\right],
$$

where the function for $\Delta \varepsilon_{\text {tot }}(\tilde{M})$ is taken in the form (8).

The engineering parameters $N^{\star}$ and $L^{\star}$ characterising creep-fatigue durability have the key importance for design applications. For ease of use, both parameters determined by Eqs (9) and (10) respectively can be represented in the form of a design contour plot, illustrated and discussed in [3].

According to the classification in R5 Volume 2/3 [5, 6] and manufacturing procedure [2], the cruciform weldment belongs to the Type 2 (Dressed). Weldments are considered to be composed of parent material and the difference in $\Delta \varepsilon_{\text {tot }}$ of the weldment compared to the parent material is taken into account by using a Fatigue Strength Reduction Factor (FSRF). The variety of FSRFs for the cruciform weldment obtained in previous works is reported in [3]. However, all these values do not take into account the influence of the dwell $\Delta t$ duration and, therefore, creep on fatigue endurance reduction. This limitation is eliminated by the application of the analytic model (9), which is transformed using Eq.(8) into the conventional form for of S-N diagrams similar to Eq.(3):

$$
\Delta \varepsilon_{\text {tot }}=p_{1}\left[a(\Delta t) / \log \left(N^{\star}\right)\right]^{1 / b(\Delta t)}+p_{2}\left[a(\Delta t) / \log \left(N^{\star}\right)\right]^{p_{3} / b(\Delta t)} \Rightarrow \operatorname{FSRF}=\frac{\Delta \varepsilon_{\mathrm{tot}}^{\text {parent }}\left(N^{\star}\right)}{\Delta \varepsilon_{\text {tot }}^{\text {x-weld }}\left(N^{\star}, \Delta t\right)},
$$

where the S-N diagram for parent material plate is defined by Eq.(3) with the following polynomial coefficients referring to [6]: $m_{0}=2.2274, m_{1}=-0.94691$ and $m_{2}=0.085943$. 


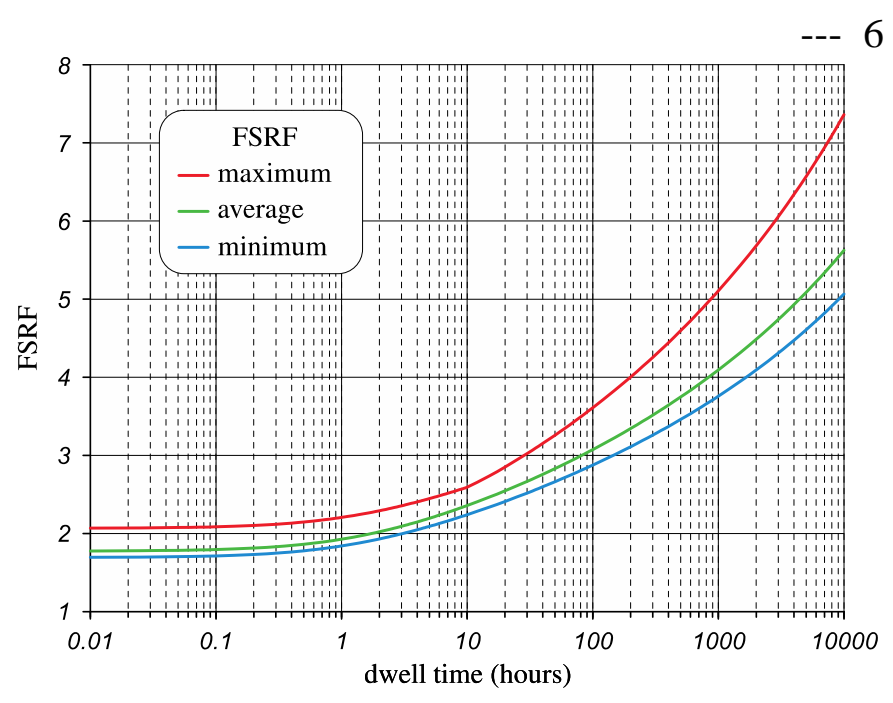

Figure 5: Dependence of FSRF on duration of dwell $\Delta t$

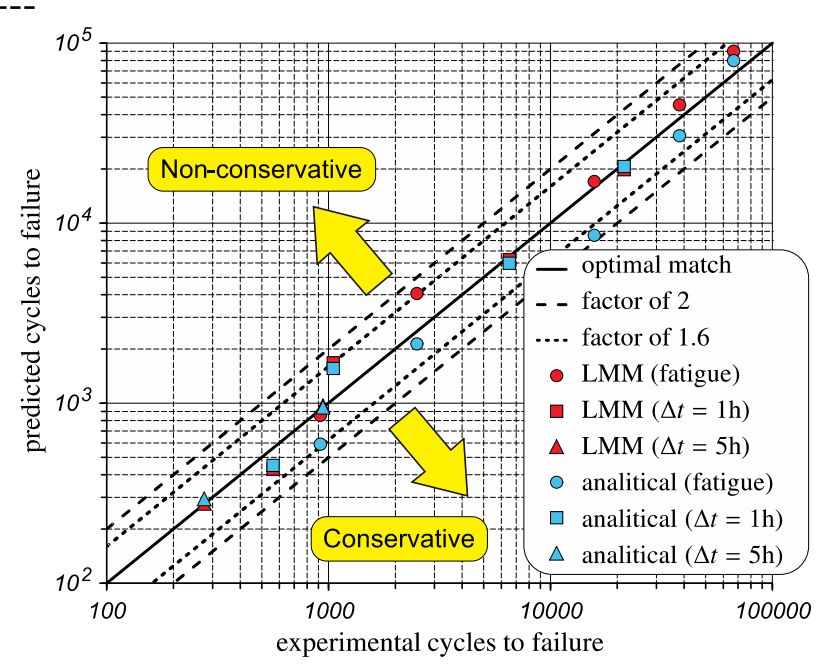

Figure 6: Comparison of the observed and predicted $N^{\star}$

The resultant dependence of FSRFs on duration of $\Delta t$ is illustrated in Fig.5, which shows significant enhancement of FSRF for $\Delta t>1$ hour caused by creep. For pure fatigue $\mathrm{FSRF}_{\min }=1.69$, $\mathrm{FSRF}_{\max }=2.06$ and average $\overline{\mathrm{FSRF}}=1.77$. Dependence of $\overline{\mathrm{FSRF}}$ on $\Delta t$ can be fitted as follows:

$$
\overline{\operatorname{FSRF}}(\Delta t)=f_{0}+f_{1} \log (\Delta t+1)+f_{2} \log (\Delta t+1)^{2}+f_{3} \log (\Delta t+1)^{3},
$$

where the fitting parameters are $f_{0}=1.7685, f_{1}=0.53422, f_{2}=0.00574$ and $f_{3}=0.02509$.

\section{Conclusions}

Comparison of the observed and predicted $N^{\star}$ with the proposed LMM-based approach for 3 types of experiments shows that simulation of 9 of 11 totally available tests is very close to the line of optimal match, as shown in Fig.6. Simulation of the other 2 experiments produces non-conservative results with an inaccuracy factor equal to 1.6, which is favourable compared to the factor of 2 allowable for engineering analysis. The proposed function for FSRF depending on dwell time $\Delta t$ (12) for Type 2 (Dressed) weldments and shown in Fig.5 allows to improve design techniques e.g. in R5 Procedure [5] by considering the significant influence of creep.

\section{References}

[1] Chen, H.F., Chen, W., Ure, J. Linear matching method on the evaluation of cyclic behaviour with creep effect. // Proc. ASME Pressure Vessels \& Piping Conf. (PVP2012-78065). Toronto, Canada: ASME; 2012, July 15-19.

[2] Bretherton, I., Knowles, G., Hayes, J.-P., Bate, S.K., Austin, C.J. PC/AGR/5087: Final report on the fatigue and creep-fatigue behaviour of welded cruciform joints. // Report for British Energy Generation Ltd no. RJCB/RD01186/R01; Serco Assurance; Warrington, UK; 2004.

[3] Gorash, Y., Chen, H.F. Creep-fatigue life assessment of cruciform weldments using the linear matching method. // Int. J. Press. Vess. Piping, 2012; 14 p., under review.

[4] Ponter, A.R.S., Chen, H.F. Modeling of the behavior of a welded joint subjected to reverse bending moment at high temperature. // J. Press. Vess. Technol., 2007; 129(2): 254-261.

[5] Ainsworth, R.A., editor. R5: An Assessment Procedure for the High Temperature Response of Structures. Procedure R5: Issue 3. // British Energy Generation Ltd, Gloucester, UK; 2003.

[6] Bate, S.K., Hayes, J.-P., Hooton, D.G., Smith, N.G. Further analyses to validate the R5 volume 2/3 procedure for the assessment of austenitic weldments. // Report for British Energy Generation Ltd no. SA/EIG/11890/R002; Serco Assurance; Warrington, UK; 2005.

[7] NIMS Creep Data Sheet No. 45A; National Institute for Materials Science; Tsukuba, Japan; 2005.

[8] Chen, H.F. Lower and upper bound shakedown analysis of structures with temperature-dependent yield stress. // J. Press. Vess. Technol., 2010; 132(1): 011202:1-8.

[9] Wada, Y., Aoto, K., Ueno, F. Creep-fatigue evaluation method for type 304 and 316FR SS. // Creep-fatigue damage rules for advanced fast reactor design. Vienna: IAEA; 1997, p. 75-86.

[10] Skelton, R.P., Gandy, D. Creep-fatigue damage accumulation and interaction diagram based on metallographic interpretation of mechanisms. // Mater. High Temp., 2008; 25(1): 27-54. 